\title{
Correction to: External Supplement of Impulsive Micromanager Trichoderma Helps in Combating CO2 Stress in Rice Grown Under FACE
}

\author{
Aradhana Mishra ${ }^{1,2} \cdot$ Satyendra Pratap Singh ${ }^{1} \cdot$ Sahil Mahfooz $^{1} \cdot$ Richa Shukla $^{1} \cdot$ Nishtha Mishra $^{1,2} \cdot$ Shipra Pandey $^{1,2}$. \\ Sanjay Dwivedi ${ }^{3}$. Vivek Pandey ${ }^{4}$. Pramod Arvind Shirke ${ }^{4} \cdot$ Chandra Shekhar Nautiyal $^{1}$
}

Published online: 18 November 2019

(C) Springer Science+Business Media, LLC, part of Springer Nature 2019

Correction to: Plant Molecular Biology Reporter (2019) 37:1-13

https://doi.org/10.1007/s11105-018-1133-8

The original version of this article unfortunately contained missing information at author's affiliations.

The affiliation address of the author's Nishtha Mishra and Shipra Pandey should include "Academy of Scientific and Innovative Research (AcSIR), Ghaziabad-201002, UP, India" as listed below.

Publisher's Note Springer Nature remains neutral with regard to jurisdictional claims in published maps and institutional affiliations.

The online version of the original article can be found at https://doi.org/ 10.1007/s11105-018-1133-8

Aradhana Mishra

mishramyco@yahoo.com

Satyendra Pratap Singh

singhsatyendra8619@gmail.com

\author{
Sahil Mahfooz \\ sahil77786@gmail.com \\ Richa Shukla \\ richa7787@gmail.com \\ Nishtha Mishra \\ nishtha0209@gmail.com \\ Shipra Pandey \\ shipra2589@gmail.com \\ Sanjay Dwivedi \\ drsdwivedi1@gmail.com
}

Vivek Pandey

v.pandey@nbri.res.in

Pramod Arvind Shirke

pashirke@nbri.res.in

Chandra Shekhar Nautiyal csnnbri@ rediffmail.com

1 Division of Plant Microbe Interactions, CSIR-National Botanical Research Institute, Rana Pratap Marg, Lucknow 226001, India

2 Academy of Scientific and Innovative Research (AcSIR), Ghaziabad, UP 201002, India

3 Division of Plant Ecology and Environmental Sciences, CSIR-National Botanical Research Institute, Rana Pratap Marg, Lucknow 226001, India

4 Plant Physiology Lab, CSIR-National Botanical Research Institute, Rana Pratap Marg, Lucknow 226001, India 